\title{
A fuzzy logic approach for highly dependable medical wearable systems
}

\author{
Cristina C. Oliveira and José Machado da Silva \\ INESC TEC \\ Faculdade de Engenharia da Universidade do Porto \\ Porto, Portugal \\ Email: cristina.oliveira@fe.up.pt, jms@fe.up.pt
}

\begin{abstract}
A new methodology for fault detection on wearable medical devices is proposed. The main strategy relies on correctly classifying the captured physiological signals, in order to distinguish whether the actual cause is a wearer health abnormality or a system functional flaw. Data fusion techniques, namely fuzzy logic, are employed to process the captured data, like the electrocardiogram and blood pressure, to increase the trust levels with which diagnostics are made. Concerning the wearer condition, additional information is provided after classifying the set of signals into normal or abnormal (e.g. arrhythmia, chest angina, and stroke). As for the monitoring system, once an abnormal situation is detected in its operation or in the sensors, a set of tests is run to check if actually the wearer shows a degradation of his health condition or if the system is reporting erroneous values.
\end{abstract}

\section{INTRODUCTION}

The advances on sensors, wireless communications and information technologies have resulted in the rapid development of various wellness or disease monitoring systems, which enable extended independent living at home and improve the quality of life. Traditionally, medicine has been based on an intervention basis (drugs, surgeries, prosthesis, etc.) to treat them. Nowadays, and regardless of the patients' age, the health care community is trying to focus on prevention and wearable monitoring systems have been proposed to meet this task.

Remote health monitoring can be used only if the monitoring device is based on a comfortable sensing interface, easy to use and customizable. Its interface must allow continuous remote control in a natural environment without interference or discomfort for the users. The textile approach to the implementation of sensing elements embedded in clothing items allows for low cost and long-term monitoring of patients and to easily customize the sensor configuration according to the needs of each individual [1]. Applying this concept it is possible to reduce health care costs maintaining the high quality of care, shift the focus of health care expenditures from treatment to prevention, provide access to health care to a larger number of patients, reduce the length of hospital stays and address the issue of specific requirements for elderly population and/or chronically ill patients.

Because these wearable monitoring systems are to be used for medical purposes (continuous monitoring, diagnosis, etc.), the reliability and safety of the system have to be perfectly controlled. Unfortunately, the complexity and the functional specificities of these systems make the existing dependability techniques developed for the aeronautics, space and automotive applications not totally appropriate for the medical field.

To overcome the lack of a dependability model that can be used for the development of complex pervasive medical monitoring devices, a fault tree analysis approach is being developed to identify the main risk of failure. A typical wearable device (hereafter the system) comprises a module to capture the biosignals, including the electrodes and the analogue front-end, a microcontroller, and a radio-frequency module to transmit data to a smartphone or personnal computer. In our approach the captured biosignals are received and analyzed within a smartphone. A rule based algorithm (fuzzy logic) decides whether these are normal or not. If not, it is diagnosed if the wearer shows an abnormal situation or instead the system is faulty. That is, a data abnormality can be due to a wearer irregular state (pathological condition or intense physical activity) or due to a degradation of the system operation.

\section{DEPENDABILITY STRATEGY}

The SIVIC system, a combined cardiac and coronary surveillance system under development (Figure 1), provides the synchronous measurement of a patient's ECG (electrocardiogram) and pressure in the abdominal aneurysm sac, in order to obtain a more robust and reliable monitoring. Biologically compatible wireless pressure sensors, which show suitable linearity and sensitivity [2], are used to capture the intra-sac aortic abdominal aneurysm (AAA) pressure and detect endoleaks. An electronic readout unit (ERU) capable of energizing the pressure sensors and capture the pressure data is placed in the chest of the patient. This unit provides also the monitoring of a 12-lead ECG using textile dry electrodes [3]. The electronic unit and the electrodes are built in a customized clothing. Data is transmitted to a smartphone for further processing, data display, and eventual communication with a healthcare center.

\section{A. Feature extraction}

The ECG conveys important hemodynamic information, such has the heart rate (HR). During an ECG cycle three 


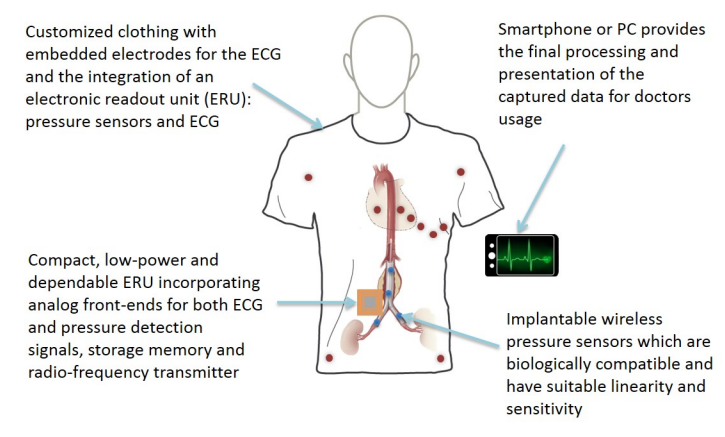

Fig. 1: Wearable ECG data capture and transmitter module.

main events take place: the $\mathrm{P}$ wave (contraction of the atria), the QRS complex (corresponding to the contraction of left ventricle) and the $\mathrm{T}$ wave (relaxation of the ventricles) (see Fig. 2). Their morphologies (amplitude and interval/segment length) will vary according to the person's physiological condition.

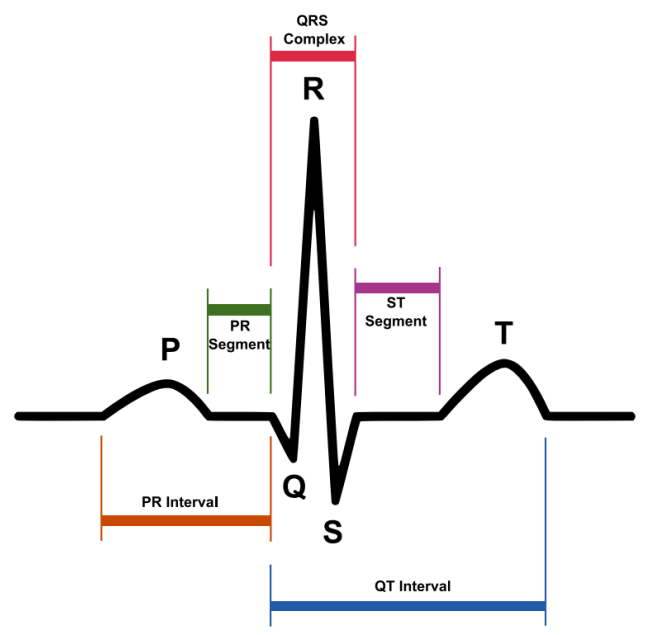

Fig. 2: Typical ECG signal and its main waves [4].

The HR is given by the counting in beats per minute (bpm) of consecutive R-waves. However, noise contamination such as baseline wander, power line interference and muscle activities can corrupt the signal and reduce the clinical value of an ECG recording. Since wearable devices are more prone to perturbations by noise, filtering of the ECG is a necessary preprocessing step to ensure a reduction of the noise components while preserving the QRS complex shape. The Pan-Tompkins algorithm is used for ECG filtering and the HR calculation [5]. Other biosignals, such as the blood pressure (BP) can provide important information about the patient condition. BP is defined by the systolic (maximum) pressure and diastolic (minimum) pressure and is measured in millimetres of mercury $(\mathrm{mmHg})$. BP is affected by the physical activity of the patient -associated diseases.

Wearable devices enable the inclusion of other sensors, such accelerometers, that enable the tracking of the wearer activity, i.e. if he is sitting, walking or running, which will influence the heart activity and thus provide useful information for the diagnosing process. The SIVIC system includes also an electrode-skin impedance measuring circuit, which enables detecting if the electrodes connected to the patient or are loose/disconnected. The main features of each signal eventually measured by the SIVIC system, the extracted features and the classification of the patient/system condition are presented in table I.

TABLE I: Data fusion model for the measured signals.

\begin{tabular}{|l|l|l|}
\hline Signals & Features & Classifier \\
\hline \hline \multirow{3}{*}{ ECG } & HR I & \\
& HR II & Normal/Abnormal \\
& HR III & \\
& $\vdots$ & \\
\hline Blood Pressure & $\begin{array}{l}\text { Systolic } \\
\text { Diastolic }\end{array}$ & Hypotensive/Normal/Hypertensive \\
\hline AAA Pressure & Mean Pressure & Normal/Endoleak \\
\hline Accelerometer & Motion & Still/Walking/Running \\
\hline $\begin{array}{l}\text { Electrode-Skin } \\
\text { Impedance }\end{array}$ & Resistance & Connected/Disconnected \\
\hline
\end{tabular}

\section{B. Fuzzy logic}

Data fusion techniques have been applied as a means for a combined analysis of several physiological signals to extract additional information on a patient's condition. Kenneth et. al performed the fusion of ECG, blood pressure, saturated oxygen content and respiratory data for achieving improved clinical diagnosis of patients in cardiac care units [6].

Fuzzy logic enables the creation of a decision making process based on logic and straightforward principles. Its implementation is relatively easy and thus suitable for implementation in a smartphone. The existing extensive literature in the medical field provides a solid knowledge base for the implementation of a medical decision support system. This technique can be applied to the monitoring of a patient's vital signs during an invasive surgery [7], support medical decisions in a intensive care unit [8], or cancer diagnosis based on image processing [9], [10].

In our case, as a first approach, a fuzzy logic system is used the data fusion due to its probability assignment based on rules. Since the values of the features extracted from the biosignals can be assigned in ranges well defined in the medical literature, the rules creation is relatively straightforward (Table II). Table II shows common normal values for the HR and BP, and some examples of pathologies.

The fuzzy logic decision system comprises 4 main components: fuzzy rules (knowledge base), fuzzy sets, fuzzy inference engine and defuzzification (Fig. 3) [11]. The inputs of the fuzzy logic system are the features previously extracted from the measured signals (Table I). The outputs are the Patient Status, System Status and the Global Status, which can be normal or faulty - i.e., either the patient has a health condition or the monitoring system is malfunctioning. The outputs are determined based on the input values of the fuzzy sets and the rules assigned for each output. The rules to 
TABLE II: Fusion rules for patient condition diagnosis.

\begin{tabular}{|c|c|c|}
\hline Signals & Condition & Rule \\
\hline \multirow{4}{*}{ ECG } & Normal & HR between 60 and $100 \mathrm{bpm}$ \\
\hline & Asystole & No QRS for at least 4 seconds \\
\hline & Extreme Bradycardia & $\begin{array}{l}\text { HR lower than } 40 \text { bpm for } 5 \text { consec- } \\
\text { utive beats }\end{array}$ \\
\hline & Extreme Tachycardia & $\begin{array}{l}\text { HR higher than } 140 \mathrm{bpm} \text { for } 17 \\
\text { consecutive beats }\end{array}$ \\
\hline \multirow{3}{*}{$\begin{array}{l}\mathrm{BP} \\
(\mathrm{mmHg})\end{array}$} & Normal & $\begin{array}{l}\text { systolic: } 90-139 \\
\text { diastolic: } 60-89\end{array}$ \\
\hline & Hypotension & $\begin{array}{l}\text { systolic: }<90 \\
\text { diastolic: }<60\end{array}$ \\
\hline & Hypertension & $\begin{array}{l}\text { systolic: }>140 \\
\text { diastolic: }>90\end{array}$ \\
\hline AAA & Normal & Low pressure $(\sim 40 \mathrm{mmHg})$ \\
\hline Pressure & Endoleak & Sistemic Pressure \\
\hline
\end{tabular}

define the Patient Status are based in information found in the literature, the rules for the System Status are defined from the system specifications, and the rules for the Global Status include both.

The fuzzy sets include the HR for each channel, the blood pressure (systolic and diastolic), and can also include the AAA pressure, the acceleration of the patient's activity, and the electrode-skin contact resistance if these data are available. The signal-to-noise ratio (SNR) of bioelectrical signals is known to be related to the electrode-skin impedance [3]. Since the impedance varies for each person and is affected by other factors like temperature and applied pressure, when the system is being used, the electrode-skin impedance is measured when it is switched on and afterwards is monitored periodically to establish a normal region for the impedance values, for which the acquired ECG quality is considered acceptable. These values are then used for comparison with the measured impedance during normal operation of the system. In case the impedance values are higher than expected, signalling a potential loose connected electrodes situation, the fuzzy logic system updates the System Status.

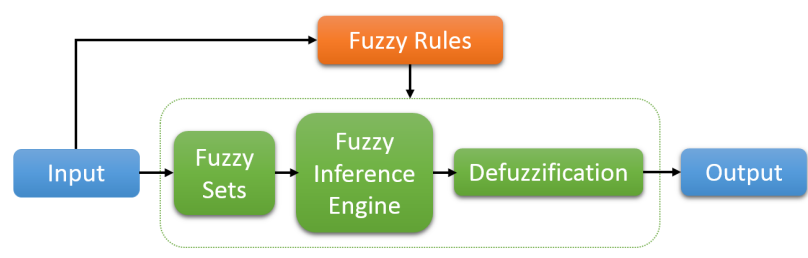

Fig. 3: Block diagram of fuzzy logic system.

The trapezoidal curve was chosen for the membership function. This is a function of a vector, $\mathrm{x}$, and depends on four scalar parameters $a, b, c$, and $d$ (equation 1). The parameters $a$ and $d$ locate the "feet" of the trapezoid and the parameters $b$ and $c$ locate the "shoulders".

$$
\mu_{\text {trapezoidal }}(x)= \begin{cases}0, & x<a \text { or } x>d \\ \frac{x-a}{b-a}, & a \leq x \leq b \\ 1, & b \leq x \leq c \\ \frac{d-x}{d-c}, & c \leq x \leq d\end{cases}
$$

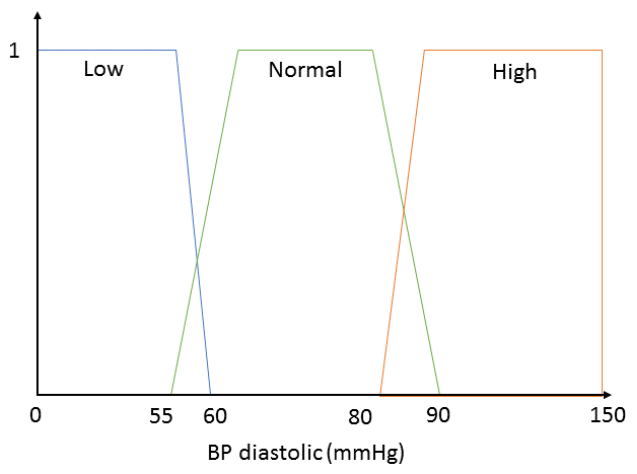

Fig. 4: Fuzzy sets for the diastolic BP.

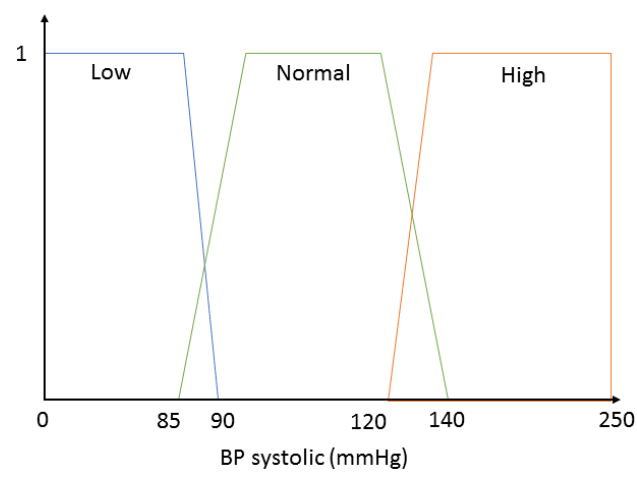

Fig. 5: Fuzzy sets for the systolic BP.

The BP is divided in three sets: low (hypotension), normal and high (hypertension). Figure 4 displays the membership functions for the diastolic BP sets and Figure 5 displays the membership functions for the systolic BP sets.

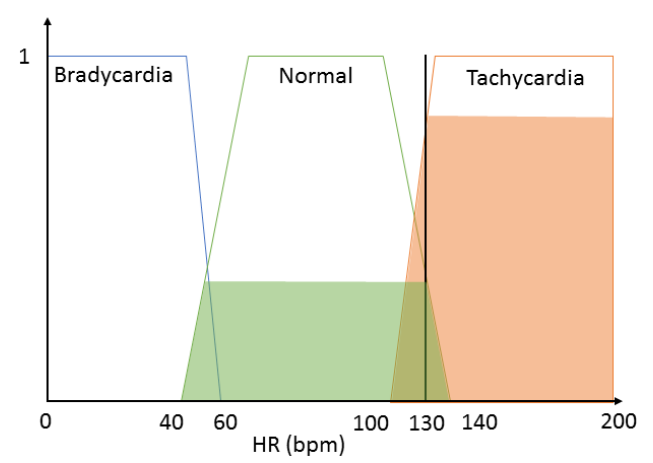

Fig. 6: Fuzzy sets for the HR.

The HR includes the following sets: bradycardia, normal and tachycardia. The vertical black line in 6 represents a HR measurement of $130 \mathrm{bpm}$, which has a membership level of 0.3 in the normal set and a level of 0.8 in the tachycardia set.

The output variables Patient Status, System Status and Global Status have 2 sets: abnormal (from 0 to 0.5 ) and normal (from 0.5 to 1 ). The normal sets from the inputs are assigned to the normal set of the outputs, and the remaining input sets are assigned to the abnormal output set. 


\section{Fault detection}

The proposed fuzzy logic system, with its three levels (Patient, System, Global) evaluation approach, is extremely useful in identifying the cause of an occurring fault. The fault tree displayed in Fig. 7 depicts the system's main components and the way how faults propagate. This high level fault tree is also utilized in the decision making process of the fuzzy logic system for fault identification purposes. When the fuzzy logic system outputs a System Status below 0.5 the smartphone requests the SIVIC system to perform the necessary tests to detect the problem source and eventually correct it. Some ECG signal disturbances and testing procedures are listed here:

- ECG signal is out of scale: check the input amplifiers by disconnecting the electrodes and connecting a known test stimulus

- ECG signal is corrupted with noise: check the electrodes impedance, test the analog front-end circuit, and the communication Bluetooth link

- QRS complex with reduced amplitude: verify the linearity of the analog front-end and the electrode-skin impedance

- Received ECG signals are corrupted

These tests are based on a Failure mode and effects analysis (FMEA) performed during the system's design. The FMEA for the implantable pressure sensors that measure the presence or absence of endoleaks in the AAA was performed and a possible testing procedure was already presented [12]. For the complete SIVIC system our analysis was based on the FMEA analysis made by [13]. This phase is very important to identify the most problematic components and functions of the system and determine which components/blocks should include builtin self-test (BIST) or other type of testing.

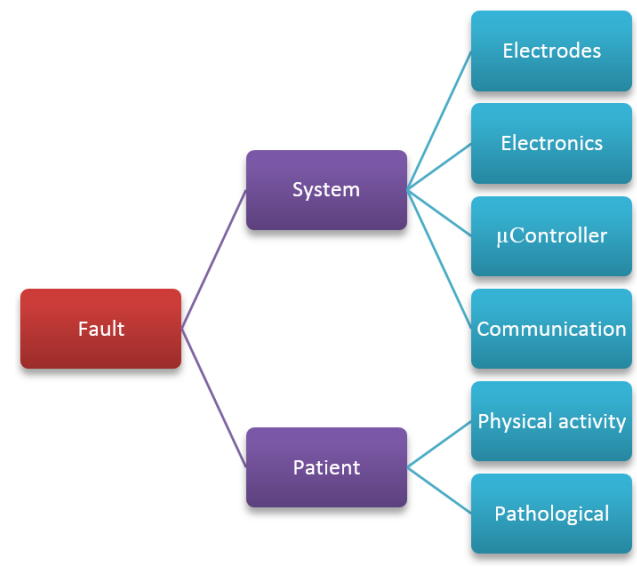

Fig. 7: Fault tree analysis of the wearable monitoring system.

\section{RESULTS AND DisCUSSION}

Data from the MIT Multiparameter database (MGH/MF) was used to evaluate the fuzzy logic system using Matlab [14], [15]. The features from ECG signals (leads I, II and V) and the arterial blood pressure (ART) were extracted and feed to the fuzzy logic system. The ECG provides the HR information and the ART waveform is used to know the systolic and diastolic pressures. Also an impedance signal was added to the set in order to test for possible faults.

The fuzzy logic was evaluated for 3 situations:

1) One of the ECG channels (lead I) does not provide useful information, most likely due to a faulty contact (Figure 8). The other channels (leads II and V) enable the detection of the HR. The electrode-skin impedance value enables detecting the problem is related with the electrode. Result: Patient Status: 81; System Status: 18; Global Status: 81.

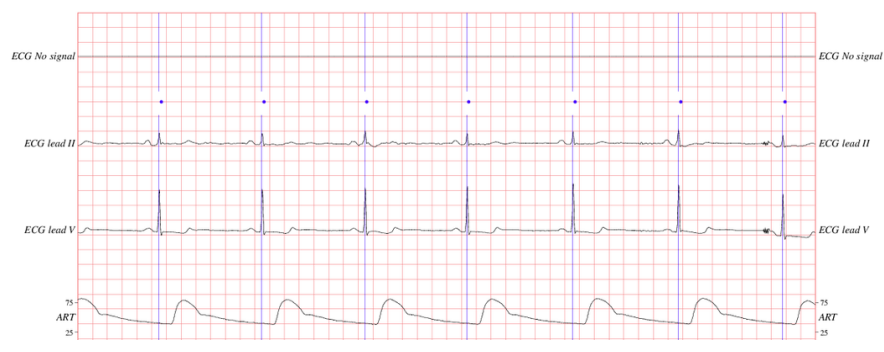

Fig. 8: MGH231 record: lead I is missing.(Grid intervals: time 0.2 $\mathrm{s}$, ECG $0.5 \mathrm{mV}$, ART $25 \mathrm{mmHg}$ )

2) The recorded signals have good quality, i.e. the SNR is good enough to identify relevant features, but the patient's blood pressure is very high (record MGH085 from the MGH/MF database). The System Status is ok, but the Patient Status indicates a health problem. Result: Patient Status: 14; System Status: 86; Global Status: 86.

3) Atrial flutter, or arrhythmia, is an abnormality of the heart rhythm resulting in a rapid and sometimes irregular heartbeat. The occurrence of atrial flutter in the ECG is recognized by the presence of characteristic flutter waves at a regular rate of 240 to 440 beats per minute (Figure 9). In this case the $\mathrm{HR}$ is calculated using lead V, and the ART waveform is also used for a more reliable HR estimation, since these signals are related. Result: Patient Status: 14; System Status: 86; Global Status: 86.

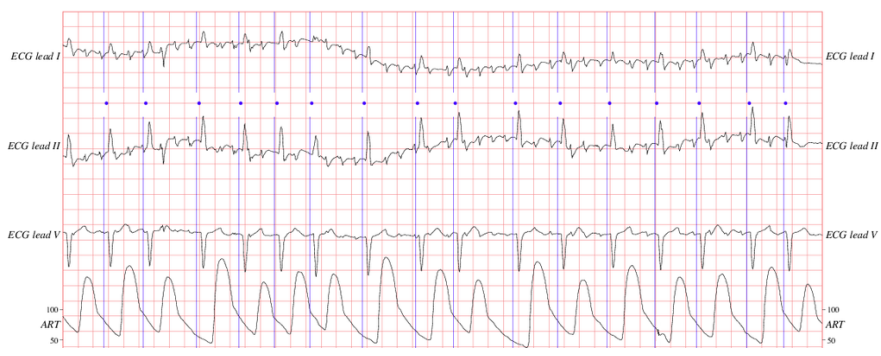

Fig. 9: MGH023 record: Atrial flutter. (Grid intervals: time $0.2 \mathrm{~s}$, ECG $0.5 \mathrm{mV}$, ART $25 \mathrm{mmHg}$ )

When the data fusion model detects that the System Status is degraded, further tests can be performed by the system to determine the cause. The smartphone sends an order for specific tests to be performed depending on the signals features. For instance if an ECG channel presents a behaviour similar to the atrial flutter condition, but the remaining channels are normal, the flutter could be caused by the acquisition system, rather 
than the patient's heart. An oscillation in the ECG amplifier could cause such flutter in the signal. A simple test would be to interconnect both inputs of the amplifier and observe if the flutter persists. If not, it is likely that the signal is really displaying a health condition that is then more visible in this particular ECG channel.

Also the data fuzzy model is flexible, in the sense that further inputs can be added to the system with extra information regarding the patient and the system. For instance, behavior identification sensors like accelerometers can be added to monitor the patient activity. If motion is detected at the same time the ECG signal is degraded, the system can determine the degradation of the biosignal as temporary and not related with any fault from the electronics or the electrodes. A body temperature sensor allows verifying if a moderately accelerated HR is due to a fever situation (the heart rate increases on average 8.5 beats per minute for a 1 degree $\mathrm{C}$ increase in body temperature [16]).

\section{CONCLUSION}

Several wearable medical monitoring systems are available, all with different architectures, components, characteristics, and designs. All authors state that high levels of reliability, security, safety, availability and maintainability are required. Such high levels of dependability are difficult to achieve due to the complexity of these monitoring systems, which have different blocks and functional layers (sensors, data acquisition front-end, software, networks, etc). A data fusion model for wearable medical systems based on fuzzy logic is presented. It is shown how fuzzy logic can be explored to correlate data obtained from different sensors in order to obtain status indicators for that characterize the operation correctness of a monitoring system or a pathological condition of the wearer.

\section{ACKNOWLEDGMENT}

This work is financed by the ERDF - European Regional Development Fund through the COMPETE Programme (operational programme for competitiveness) and by National Funds through the FCT - Fundação para a Ciência e a Tecnologia (Portuguese Foundation for Science and Technology) within project SIVIC PTDC/EEI-ELC/1838/2012 (FCOMP-01-0124FEDER-028937), and grant contract SFRH/BD/81476/2011 (first author).

\section{REFERENCES}

[1] R. Paradiso, G. Loriga, and N. Taccini, "A wearable health care system based on knitted integrated sensors," Information Technology in Biomedicine, IEEE Transactions on, vol. 9, no. 3, pp. 337-344, 2005.

[2] C. Oliveira, A. Sepúlveda, N. Almeida, B. Wardle, J. Machado da Silva, and L. Rocha, "Implantable flexible pressure measurement system based on inductive coupling," Biomedical Engineering, IEEE Transactions on, vol. 62, no. 2, pp. 680-687, Feb 2015.

[3] C. Oliveira, J. Machado da Silva, I. Trindade, and F. Martins, "Characterization of the electrode-skin impedance of textile electrodes," in 2014 Conference on Design of Circuits and Integrated Circuits (DCIS), Nov. 2014, pp. 1-6.

[4] M. Elgendi, B. Eskofier, S. Dokos, and D. Abbott, "Revisiting QRS Detection Methodologies for Portable, Wearable, Battery-Operated, and Wireless ECG Systems," PLOS ONE, vol. 9, no. 1, p. e84018, Jan. 2014. [Online]. Available: http://dx.doi.org/10.1371/journal.pone.0084018
[5] J. Pan and W. J. Tompkins, "A Real-Time QRS Detection Algorithm," IEEE Transactions on Biomedical Engineering, vol. BME-32, no. 3, pp. 230-236, Mar. 1985.

[6] E. Kenneth, A. U. Rajendra, N. Kannathal, and C. M. Lim, "Data fusion of multimodal cardiovascular signals," in Engineering in Medicine and Biology Society, 2005. IEEE-EMBS 2005. 27th Annual International Conference of the, 2005, pp. $4689-4692$.

[7] K. Becker, B. Thull, H. Ksmacher-Leidinger, J. Stemmer, G. Rau, G. Kalff, and H.-J. Zimmermann, "Design and validation of an intelligent patient monitoring and alarm system based on a fuzzy logic process model," Artificial Intelligence in Medicine, vol. 11, no. 1, pp. 33-53, Sep. 1997. [Online]. Available: http://www.sciencedirect.com/science/article/pii/S0933365797000201

[8] J. H. T. Bates and M. P. Young, "Applying Fuzzy Logic to Medical Decision Making in the Intensive Care Unit," American Journal of Respiratory and Critical Care Medicine, vol. 167, no. 7, pp. 948-952, Apr. 2003. [Online]. Available: http://www.atsjournals.org/doi/full/10.1164/rccm.200207-777CP

[9] M. Mahfouf, M. F. Abbod, and D. A. Linkens, "A survey of fuzzy logic monitoring and control utilisation in medicine," Artificial Intelligence in Medicine, vol. 21, no. 13, pp. 27-42, Jan. 2001. [Online]. Available: http://www.sciencedirect.com/science/article/pii/S0933365700000725

[10] M. F. Abbod, D. G. von Keyserlingk, D. A. Linkens, and M. Mahfouf, "Survey of utilisation of fuzzy technology in Medicine and Healthcare," Fuzzy Sets and Systems, vol. 120, no. 2, pp. 331-349, Jun. 2001. [Online]. Available: http://www.sciencedirect.com/science/article/pii/S0165011499001487

[11] L. A. Zadeh, "Fuzzy Logic," Computer, vol. 21, no. 4, pp. 83-93, Apr. 1988.

[12] C. Oliveira and J. da Silva, "Fault Detection System for a Stent-Graft Endoleakage Monitor," in Mixed-Signals, Sensors and Systems Test Workshop (IMS3TW), 2012 18th International, 2012, pp. 17-21.

[13] M. Cinque, A. Coronato, and A. Testa, "Dependable Services for Mobile Health Monitoring Systems," Int. J. Ambient Comput. Intell., vol. 4, no. 1, pp. 1-15, Jan. 2012. [Online]. Available: http://dx.doi.org/10.4018/jaci.2012010101

[14] A. L. Goldberger, L. A. N. Amaral, L. Glass, J. M. Hausdorff, P. C. Ivanov, R. G. Mark, J. E. Mietus, G. B. Moody, C.-K. Peng, and H. E. Stanley, "PhysioBank, PhysioToolkit, and PhysioNet Components of a New Research Resource for Complex Physiologic Signals," Circulation, vol. 101, no. 23, pp. e215-e220, Jun. 2000. [Online]. Available: http://circ.ahajournals.org/content/101/23/e215

[15] J. Welch, P. Ford, R. Teplick, and R. Rubsamen, "The massachusetts general hospital-marquette foundation hemodynamic and electrocardiographic database - comprehensive collection of critical care waveforms," J Clinical Monitoring, vol. 7, no. 1, pp. 96-97, 1991.

[16] V. M. Karjalainen J, "Fever and cardiac rhythm," Arch Intern Med., vol. 146, no. 6, pp. 1169-71, Jun. 1986. [Online]. Available: http://dx.doi.org/10.4018/jaci.2012010101 